\title{
Artistic brain-computer interfaces: state-of-the-art control mechanisms
}

\author{
Amy Wadeson $^{\mathrm{a}}$, Anton Nijholt ${ }^{\mathrm{b}}$ (I) and Chang S. Nam ${ }^{\mathrm{a} *}$ \\ ${ }^{a}$ Edward P. Fitts Department of Industrial and Systems Engineering, North Carolina State University, Raleigh, NC 27695, USA; \\ ${ }^{b}$ Human Media Interaction, University of Twente, Enschede, The Netherlands
}

(Received 23 September 2015; accepted 30 September 2015)

\begin{abstract}
Artistic BCI applications offer a new modality for humans to express themselves creatively. In this survey we reviewed the available literature on artistic BCIs by classifying four types of user control afforded by the available applications: selective control, passive control, direct control and collaborative control. A brief overview of the history of artistic BCIs is presented, followed by examples of current artistic BCI applications in each defined sector of control. We questioned whether or not creative control affects the users' sense of enjoyment or satisfaction. Finally, we made suggestions for the future of artistic BCI research to question the role that control plays in user satisfaction and entertainment.
\end{abstract}

Keywords: brain-computer interface; artistic; music; creative; control

\section{Introduction}

Artistic mediums have recently moved beyond musical instruments, paintbrush and paper, and computer drawing tablets into the realm of artistic brain-computer interfaces (BCIs). Artistic BCIs are applications in which users employ their brainwaves to manipulate a physical or (digital) audiovisual environment resulting in a creative work of music, art, dance, or writing. Although conventional artistic computer interfaces continue to be more reliable and efficient particularly among healthy individuals,[1] artistic BCIs have opened the door for the severely disabled to communicate and create. Art and music therapy are now a reality for those suffering from locked-in syndrome, amyotrophic lateral sclerosis (ALS), or spinal cord injury.

Artists have also explored the possibilities of BCIs to create new art forms and integrate BCIs into their interactive audiovisual and physical installations.[2] An example of BCI and physical artistic integration is found in Christoph De Boeck's 'Staalhemel', or Steel Sky.[3] Staalhemel is an interactive musical BCI that maps users' brainwaves onto the playing of steel squares that are suspended from the ceiling. When users are in a relaxed state, their alpha waves $(7-13 \mathrm{~Hz})$ are transmitted and the steel sky is silent, other than one 'ding' every four seconds. When users are focused, beta rhythms $(13-30 \mathrm{~Hz})$ are present and the steel sky lights up with cacophonous sounds of banging steel. The musical feedback prompts the user to either continue in their same mental state, or to attempt to change their current brainwave frequencies, depending on personal preference.
Similar to most artistic mediums, different BCI applications allow for varying amounts of creative control (see Table 1). Staalhemel would be considered a 'selective control' $\mathrm{BCI}$, as it allows for users to interact and attempt to control their brainwaves to change the musical landscape, but it does not give them complete creative freedom. That is, users cannot look at a steel square on the ceiling and intentionally make only that square play, or they cannot create a specific rhythm on their own accord. They can create unique and individualized music through controlling their level of focus or relaxation, however, which gives them selective control over the artistic environment.

Many artistic BCIs limit users to more of a 'passive control' state. These types of artistic BCI applications 'listen' to the brainwaves of users, and then a computer maps those signals onto notes or other artistic information. This is done without much active participation on behalf of the user, meaning that users are not asked to interact with any interface, or make any selections. The system simply interprets brainwaves and outputs pre-programmed artistic material. Artistic BCIs that afford users more 'direct control' over the physical or audiovisual environment also exist. In general, these applications are considered more of a toolbox akin to MS Paint, which allows users to make intentional selections and draw what they want. Limitations still occur, however, due to current BCI technological capabilities. Collaborative effort is not uncommon in the art world and thus certain artistic BCIs have also attempted to allow cooperation among users to create music or digital art. The Multimodal Brain Orchestra (MBO) [4] is one example of a 'collaborative control' musical BCI. A 'unique aspect of the $\mathrm{MBO}$ is that it

\footnotetext{
*Corresponding author. Email: csnam@ncsu.edu 
Table 1. Summary of four main control types in artistic BCIs.

\begin{tabular}{lll}
\hline Control type & Definition & $\begin{array}{l}\text { Example } \\
\text { references }\end{array}$ \\
\hline Passive & $\begin{array}{l}\text { Heavily reliant on pre-programmed artistic material. These systems are built to respond to certain } \\
\text { brainwave signals, which do not require interaction or intention from the user in order to create the } \\
\text { desired signals. }\end{array}$ \\
Allow for interaction of user by way of controlling emotion, levels of relaxation or excitement, etc. \\
to affect the end artistic result. However, user not directly responsible for the output, due to the \\
application relying on pre-programmed artistic material.
\end{tabular}

allows for a multimodal and collaborative performance involving four brain orchestra members, a musical conductor and real-time visualization' (p. 309). Through the use of SSVEP and P300 BCIs the orchestra players affect the musical environment while also taking commands from the conductor, who wields a Wii remote that interacts with the system. All BCI applications require brain signals to be translated into 'commands' that affect a physical or (digital) audiovisual environment. These commands can be given explicitly and meaningfully from the user, or they can originate from monitoring intentional or unintentional changes in brainwave activity. The different types of commands and the ways in which user(s) interact with the application define our definitions of control: passive, selective, direct, and collaborative. The separation of artistic BCIs into control categories is a novel way of comparing different BCI applications and research. This is helpful for novices and researchers alike, as recent interest in learning about, improving, and inventing artistic BCIs has increased significantly. This paper gives structure to an ever-evolving field and gives a brief overview of each type of BCI control, allowing future researchers a quick look into relevant resources as they decide which type of artistic BCI they would like to research or create.

In the rest of this paper we present a brief history of artistic brain-computer interfaces, discuss a sampling of artistic BCIs that exemplify our definitions of control mechanisms, and conclude with a discussion on control and the role it plays in user satisfaction.

\section{History of artistic BCIs}

Measured electrical activity in the brain has been used for artistic performances since the 1960s. Music performances were done by Alvin Lucier in the USA in 1965, [5] Richard Teitelbaum in Italy in 1967 and Pierre Henry and Roger LaFosse in France in 1971. Nina Sobell introduced a Brainwave Drawing Game in the early 1970s.
Jacqueline Humbert introduced Brainwave Etch-a-Sketch in 1974. Many of these early applications can be found in David Rosenboom's book 'Biofeedback and the Arts'.[6] During these years there was limited knowledge about human brainwaves. Joe Kamiya's paper on conscious control of brainwaves (alpha waves) appeared in 1968.[7] Jacques Vidal's paper in which BCI was introduced appeared in 1973.[8] At that time, Vidal mentioned, 'All these methods are geared to a type of computer procedure known as batch processing, where data sets are created during an experiment and analyzed later. None of them is readily amenable to the interactive, on-line, real-time feature extraction' (p. 169).

It is not our aim here to give a comprehensive survey of all artistic applications that use EEG input and that have been developed since then. The obvious difference with these very early years is the availability of computer power to store and manipulate information, progress in neuroscience, the ever decreasing costs of computing technology, the emergence of multimedia, and, in recent years, the decreasing costs of BCI technology. We now have the capability to sonificate and visualize brain signals, to map them onto musical or animation parameters, to use them to synthesize new works of art or modify existing works of art, and to control musical or painting toolboxes and instruments using various BCI techniques. Various papers sketch the development from the early artistic applications with EEG input to the fully fledged artistic BCI applications that we can find today. $[9,10]$ In [2] many examples of recent BCI applications in the musical domain can be found.

\section{BCI control paradigms}

\subsection{Passive control in artistic BCIs}

When discussing issues of control across artistic BCIs, we start at the lowest level with passive control. Passive control BCIs are identified by applications that are more heavily reliant on computer systems than on human 
interaction with the computer system. The system receives input from a non-interactive user, interprets the signals and outputs artistic material based on the parameters that have been programmed.

The BCMI Piano is an example of a passive control artistic BCI.[11] Miranda and his colleagues created a computer program that interprets brain signals from a silent and non-interactive user. The EEG headset is worn by the user and with each brainwave signal interpretation the program makes a decision on what music to play. Signal complexity helps to further progress the music by influencing tempo and volume. 'The BCMI piano implements a generative system that composes music using rules extracted from a given corpus of examples. It extracts sequencing rules from the examples and creates a transition matrix representing the transition-logic of what-follows-what' (p. 2). As the user is not intentionally interacting with the BCI, this system falls under our definition of 'passive control'.

Another passive control example from Miranda [12] is his EEG Melodies program. This is a musical BCI that analyzed signals from individual electrodes on the EEG and arbitrarily mapped each electrode to a note. Each time an electrode was activated a note was played. Users were not expected to actively participate in the signal creation by interacting or responding to any sort of stimulus. Therefore, this BCI also falls under our definition of a passive control artistic BCI.

An example of an offline, passive BCI is found in [13]. EEG data were collected, pre-processed, and then musical outputs were applied to different signal parameters. For example, the duration of a note was determined by the EEG event period, the pitch was chosen through amplitude, and the intensity was obtained through change in EEG energy (p. 2). Through these assignments of brain signal data to musical information, a new and more complex way of turning brainwaves into music emerged. The experimenters also questioned the difference in eyes-open music and eyes-shut music. Interestingly, and probably expectedly, eyes-closed music was slower, longer, and lower pitched, than eyesopen music. Again, participants in this experiment were not expected to interact with any interface and their data were transformed into music after they were collected, not in real time, thus exemplifying a passive artistic BCI.

\subsection{Selective control in artistic BCIs}

Selective control BCIs, as per our definition for this paper, allow users to interact with the artistic BCI environment, though certain outcomes may be unintentional or involuntary - such as the music that is played by [3]. We have identified two types of passive control artistic BCIs: those that require brainwave control and those that require random selection through SSVEP or P300 interfaces, both of which result in output that is not strictly created by the user.

Examples of selective control artistic BCIs include much of the work presented by Miranda and his colleagues.[11,12,14,15] In [11] Miranda discusses the BCMI audio mixer, which allows users to control the volume of certain segments in a pre-composed musical piece by controlling their alpha and beta brainwaves. Although users have control over which part of the composition's volume is lowered or raised, the users have not composed the piece themselves. Therefore, we consider Miranda's musical BCI to grant the user selective control using brainwave frequency modulation.

Flex, a musical BCI implemented in [14], gives users some control over the musical output of the system.

The user is presented with four on-screen options that flash at different frequencies in order to obtain SSVEP stimuli in the EEG signal. Each time the user focuses on one of the visual options, the signal is sent through the EEG, interpreted by the computer, and sounds are then output. The computer system then changes the sounds that are associated with each option, and the user has to figure out which selection to make next to follow the proper composition. If the user tries to select the same option too much, the computer will change that overly selected icon to produce lower or louder sounds, instead of new material. The user responds to the feedback of the system by choosing new buttons to try and regain proper control over the composition. Due to the user involvement in the selection of visual stimuli, though not in the programming of the actual sounds, and given that control is given and taken away by the computer program, this BCI falls into the selective control category that requires random selection.

Todd et al. [16] used a steady-state evoked potential (SSVEP) based BCI for a drawing application. A computer screen surrounded on top, bottom, and both sides by four LED lights was set up. Each light was associated with a function, and the participants were asked to focus on the light associated with the function they wanted to perform. Within the drawing segment of their application the LED lights were associated with different shapes. Users selected as many shapes as they'd like within a given time period. Due to the limited number of options with the LED lights, participants were only able to choose which shape they wanted and the computer decided on the color and placement of each selection. So, although users had control over which shapes and how many were placed on the digital canvas, they were only granted selective control because of the limitation on placement and color. 


\subsection{Direct control in artistic BCIs}

Our definition of a direct control artistic BCI is one in which users are able to conceptualize a song or drawing and use the tool box provided to attempt to make this conceptualization a reality. Though many of these toolbox-style BCIs are limited due to current available technology, they still allow users to make conscious, intentional decisions about what exactly they would like the system to output. BCI control can be done by using BCI paradigms such as P300 or SSVEP, but we can as well think of motor imagery to go up and down on a menu or manipulate alpha activity in order to make choices. As a side note, perfect (real-time) control cannot be expected with any form of BCI, thus limiting the control of the user further.

Whether such a BCI toolbox is used to play a particular instrument, to compose a piece of music, or to make a painting, we can say that it is mainly the user of the $\mathrm{BCI}$ toolbox that is responsible for the result, rather than the programmer of the toolbox. But obviously, the toolbox can be accompanied by a database of examples, predefined musical fragments, figures and animations and there can be built-in suggestions or restrictions on how to use the toolbox and how to combine and integrate the elements of the databases in order to obey certain 'laws' of artistic expression. A toolbox can have settings that guide the user to Pollock, Mondrian or Keith Haring-like paintings, or to Satie or Philip Glass-like compositions. Hence, the toolbox has been invaded by artistic input. The artistic expression is a joint result of a user (artist or dilettante) using the toolbox and a rule-based or algorithmic interpretation of the style of a particular artist. The $\mathrm{BCI}$ user is in control as far as accuracy of interpretation of brain signals allows.

Chew et al. [17] created a BCI for users to generate music in real time, allowing them to pick a note, listen to the selection, and then choose their next note. They used a modified P300-speller interface with an 8 by 8 matrix for a total of 64 note choices. Users were allowed to make whatever note selection they liked, giving them control over the composition of their musical piece.

The limiting factors of control in this BCI are found in the lack of choice of instrument, or note duration. That being said, the level of control afforded by this $\mathrm{BCI}$ is more direct in that users are intentionally making decisions on what precise note they would like to hear the BCI application play. Münßinger et al. [18] produced an artistic BCI application that allows users to paint by using a modified P300-speller interface equipped with a $6 \times 8$ matrix including choices such as color, object, size, transparency, zoom, and cursor movement. These options gave users the freedom to create a painting of their choice without being overly limited. For further informa- tion on the usability of this program by users with ALS, look to [19]. Zickler et al. [20] created an artistic, multimodal BCI that would appeal to healthy individuals, and could still potentially be used by individuals who were paralyzed below the neck. They hypothesized that in order for able-bodied users to want to use a BCI over conventional artistic computer interfaces their application would need to be reliable, accurate, and user-friendly. Owing to the lower level of movement restriction in healthy individuals, they were able to incorporate a modified P300-speller interface for selection of different brushes and colors, head movements for control of the brush on the digital canvas, and eye blinking to turn the brush on and off. Users had control over the digital canvas using the toolbox provided in the application.

\subsection{Collaborative control in artistic BCIs}

Collaborative control BCIs are those that are operated by more than one user. The Multi Brain Orchestra,[4] as mentioned above, is one such type of collaborative control BCI. Another example is found in [21]. This musical $\mathrm{BCI}$ is not only collaborative in the employment of many users, but also in the combination of BCI and live musical instrument performance.

It is also somewhat of a passive BCI in that musicians respond to the firing of neurons, but the neurons are not intentionally fired through stimulus response or brainwave control. The pre-composed music is performed by an orchestra. Each member of the orchestra is hooked up to an EEG system that receives signals of spiking neuronal networks. With each spiking, a LED light flashes to different orchestra members, who have been previously instructed to perform a certain musical variation every time they are presented with the LED stimulus. Pre-composed music is thus integrated with BCI stimulated music, creating a unique piece each time it is played.

\section{Discussion}

This paper has defined control as the amount of intentional activity and choice in a given application. But what do users need in order to feel that they are in control? Do users require creative freedom, a large number of selection options, high accuracy levels or a combination of these things to feel that they are in control of a system? More important, however, is understanding whether or not control is a necessary factor in users feeling satisfied or entertained, as artistic BCIs are partially considered for their entertainment value. Levels of control necessary for satisfaction or entertainment may vary for each type of user group. As reported in [18], disabled participants reported greater enjoyment of the tasks than their healthy counterparts, despite their accuracies being 
lower. If accuracy is an indicator of control, varying degrees of control may be necessary for different users to feel satisfied or entertained. This discrepancy in enjoyment and accuracies between disabled and healthy participants may be due to factors such as expectations or amount of access to creative outlets. Disabled users may enjoy the artistic BCI applications more because they may have limited opportunities to express themselves creatively.

Van de Laar et al. [22] created an experimental game environment in order to investigate whether users' accuracies and sense of fun correlated with their sense of control. Within each round of the game, users were afforded different levels of control. Users had the most control when the game did exactly as the user intended and the least control when the game only did as they intended part of the time. They found that as the game allowed for greater control the users reported a perceived increase in control. That is, the more the game responded correctly to their inputs, the more control the users felt they had over the game. They also found that "control is needed for fun, up to a certain point, after which fun decreases' (p. 7), speculating that boredom kicks in when the control level is too high due to a lack of challenge. These results can help shape the future of artistic BCIs by leading researchers to question whether accuracies matter as much for users to have fun while using artistic applications as they do for games. Furthermore, future research could address factors that affect users' sense of control while participating in artistic activities and how those factors can help shape the future of artistic BCI applications.

Emotion is such a large part of the creative process yet not many emotional, artistic BCIs have been researched or created. Makeig et al. [23] performed a collaborative, musical, emotional $\mathrm{BCI}$ to an audience of brain researchers. A participant was brought in for multiple training sessions in which he associated certain sounds with certain emotions, and a computer classified those emotions and connected them with the related sounds. Musicians were trained to recognize each sound and play an associated medley upon hearing it. During the live performance, the participant listened for an emotional cue, and then re-imagined the same feelings he had while performing the training operations. The sound associated with that feeling then played, and the musicians recognized that sound and played the accompanying music. This study shows that emotions can be categorized in brainwave signals, which could make the future of artistic BCIs extremely interesting. When an author or painter creates a piece of art, whether it is a novel or a masterpiece, their emotions have an effect on the outcome of the piece. This effect may be intentional, in that the artist sat down to create something peaceful, or angry, or sad, or ecstatic, or it can be subtly noted in the undertones of the writing or the brush strokes. Future artistic BCIs could modify menu choices based on the perceived mood of the user (direct control), or automatically add some artistic changes (selective or passive control). While this shift may seemingly remove some control from the user, it could also support the user in providing more appropriate options or outputs to meet their current emotional state. Changing the control options or the output based on mood could also offer the user an opportunity to gain further control by shifting emotional states when desired to change the outcome of the art. The idea of shifting emotional states to gain control over a BCI is also found in [24], where the authors present a game called alphaWorld of Warcraft (alphaWoW). AlphaWoW is a hybrid BCI that uses a participant's emotional state (relaxed or focused, which are correlated with alpha and beta brainwaves) to command a small portion of the game. The commands change a user's character's form (e.g. from elf while outputting alpha waves to bear while outputting beta waves), and the user has to try and shift into the proper brain state in order to play in the form that they want. Initially, this game appears to be a passive control game, in that the computer interprets the signal without much intentional effort on the part of the user. However, once a command has been interpreted to change the form of the character, the user becomes more involved in trying to regain control over their character and brainwave output (thus shifting to a direct control BCI). It could be difficult for the user to remain in a calm state (alpha) while in a highstress gaming situation, potentially causing the user to have to adapt to the game scenario by playing in a character form that she doesn't prefer until she can regain her sense of calm and shift back to outputting alpha waves. This study can help shape future research into artistic BCIs for therapy purposes, helping patients work through anger or fear by teaching them to focus on relaxing their emotional state in order to create different types of art. Researchers can look to [25] for more information on emotional BCIs.

Unlike conventional computer interfaces in which keyboard entry can directly affect computer output, BCIs are limited by their input signal (usually through an EEG), which is generally cluttered with extra noise and signals that are irrelevant for the input. Fortunately, technology has advanced so far as to allow healthy individuals to use certain BCIs with consistent, high-level accuracies with only a small amount of training.[26] Individuals with disabilities, especially those with degenerative diseases, potentially have the obstacle of less recordable brain function, a factor that could create difficulties in maintaining high accuracy levels as the system has a heavier work load in interpreting the input. BCI limitations should be considered in determining how much control a user can have over an application. 


\section{Conclusion}

Our paper took a control approach to sorting current artistic BCIs into passive, selective, direct, and collaborative control categories. Each application discussed plays a unique and important role in shaping the future of artistic BCIs. Forthcoming research should consider the role of control in users' feelings of satisfaction and entertainment in the design of their artistic BCIs.

\section{Acknowledgement}

This work was supported in part by the National Science Foundation (NSF) under Grant IIS-1421948. Any opinions, findings, and conclusions or recommendations expressed in this material are those of the authors and do not necessarily reflect the views of the National Science Foundation.

\section{Disclosure statement}

No potential conflict of interest was reported by the authors.

\section{Funding}

This work was supported by the National Science Foundation [grant number IIS-1421948].

\section{ORCID}

Anton Nijholt (D) http://orcid.org/0000-0002-5669-9290

\section{References}

[1] Gürkök H, Nijholt A. Affective brain-computer interfaces for arts. In: Affective Computing and Intelligent Interaction (ACII); 2013 Humaine Association Conference on (pp. 827-831). IEEE.

[2] Mullen T, Khalil A, Ward T. MindMusic: playful and social installations at the interface between music and the brain. In: Nijholt A, editor. Gaming media and social effects. Singapore: Springer; 2015. More Playful User Interfaces. p. 197-229.

[3] Staalhemel: responsive environment for brainwaves [Internet]. Available from: http://www.staalhemel.com/

[4] Le Groux S, Manzolli J, Verschure PF, et al. Disembodied and Collaborative Musical Interaction in the Multimodal Brain Orchestra. Proc Int Conf New Interfaces Musical Expression. 2010;309-314.

[5] Lucier A. Music for Solo Performer [Internet]. Available from: https://www.youtube.com/watch?v=bIPU2ynqy2Y

[6] Rosenboom D, editor. Biofeedback and the Arts: results of early experiments. Vancouver: Frog Peak Music; 1976.

[7] Kamiya J. Conscious control of brainwaves. Psych Tod. 1968;1:56-60.

[8] Vidal J. Toward direct brain-computer communication. Ann Rev Biophy Bioeng. 1973;2:157-180.

[9] Brouse A. A young person's guide to brainwave music. HorizonZero: Digital Art + Culture; 2004.

[10] Haill L. ICT \& Art Connect: Revelations by Flicker, Dreamachines and Electroencephalographic Signals in Art.
Proceedings Conference: AISB50, At Goldsmiths University, Volume: The Future of Art and Computing: A PostTuring Centennial Perspective; 2014.

[11] Miranda, E. R, Durrant, S, Anders, T. Towards brain-computer music interfaces: progress and challenges. In: Applied Sciences on Biomedical and Communication Technologies; 2008, October. ISABEL'08. First International Symposium on (pp. 1-5). IEEE; 2008.

[12] Miranda ER. Plymouth brain-computer music interfacing project: from EEG audio mixers to composition informed by cognitive neuroscience. Int J Arts Tech. 2010;3:154-176.

[13] Wu D, Li C, Dezhong Y. Scale-free brain quartet: artistic filtering of multi-channel brainwave music. PLoS ONE. 2013;1-7.

[14] Eaton, J, Miranda, E. BCMI Systems for Musical Performance. In: 10th International Symposium on Computer Music Multidisciplinary Research (CMMR): Sound, Music and Motion; 2013

[15] Eaton J, Miranda ER. Real-time notation using brainwave control. Proc Sound Music Comp Conf, SMC. 2013.

[16] Todd, D. A., McCullagh, P. J., Mulvenna, M. D., Lightbody, G. Investigating the use of brain-computer interaction to facilitate creativity. In: Proceedings of the 3rd Augmented Human International Conference (p. 19); 2012, March. ACM.

[17] Chew, Y. C. D., Caspary, E. MusEEGk: a brain computer musical interface. In: CHI'11 Extended Abstracts on Human Factors in Computing Systems (pp. 1417-1422); 2011, May. ACM.

[18] Münßinger J, Halder S, Kleih S, et al. Brain painting: first evaluation of a new brain-computer interface application with ALS-patients and healthy volunteers. Front Neurosci. 2010;4.

[19] Zickler C, Halder S, Kleih S, et al. Brain painting: usability testing according to the user-centered design in end users with severe motor paralysis. Art Intell Med. 2013;59:99-110.

[20] van de Laar, B. L. A., Brugman, I., Nijboer, F. Poel, M. Nijholt, A. BrainBrush, a multimodal application for creative expressivity. In: Sixth International Conference on Advances in Computer-Human Interactions (ACHI 2013); 24 Febr - 01 Mar 2013; Nice, France. pp. 62-67. IARIA XPS Press. ISBN 978-1-61208-250-9; 2013

[21] Matthias J, Ryan N. Cortical songs: musical performance events triggered by artificial spiking neurons. Body Space Tech J. 2007.

[22] van de Laar B, Plass-Oude Bos D, Reuderink B, et al. How much control is enough? Influence of unreliable input on user experience. IEEE Trans Cybernet. 2013;43:1584-1592.

[23] Makeig S, Leslie G, Mullen T, et al. First Demonstration of a Musical Emotion BCI. In: D'Mllo S, et al. editors. ACII, Part II. 2011;487-496.

[24] Plass-Oude Bos D, Reuderink B, van de Laar B, et al. Brain-computer interfacing and games. In: Tan DS, Nijholt A, editors. Brain-computer interfaces, human-computer interaction series. London: Springer-Verlag; 2010. p. 149178.

[25] Molina GG, Tsoneva T, Nijholt A. Emotional Brain-Computer Interfaces. IEEE. 2009;6:9-25.

[26] Guger C, Daban S, Sellers E, et al. How many people are able to control a P300-based brain-computer interface (BCI)? Nuero Letters. 2009;462:94-98. 